\title{
Parameter sensitivity study of a Field II multilayer transducer model on a convex transducer
}

Bæk, David; Jensen, Jørgen Arendt; Willatzen, Morten

Published in:

2009 IEEE International Ultrasonics Symposium (IUS)

Link to article, DOI:

10.1109/ULTSYM.2009.5441669

Publication date:

2009

Document Version

Publisher's PDF, also known as Version of record

Link back to DTU Orbit

Citation (APA):

Bæk, D., Jensen, J. A., \& Willatzen, M. (2009). Parameter sensitivity study of a Field II multilayer transducer model on a convex transducer. In 2009 IEEE International Ultrasonics Symposium (IUS) IEEE.

https://doi.org/10.1109/ULTSYM.2009.5441669

\section{General rights}

Copyright and moral rights for the publications made accessible in the public portal are retained by the authors and/or other copyright owners and it is a condition of accessing publications that users recognise and abide by the legal requirements associated with these rights.

- Users may download and print one copy of any publication from the public portal for the purpose of private study or research.

- You may not further distribute the material or use it for any profit-making activity or commercial gain

- You may freely distribute the URL identifying the publication in the public portal 


\title{
Parameter sensitivity study of a Field II multilayer transducer model on a convex transducer
}

\author{
David Bæk*, Jørgen Arendt Jensen*, and Morten Willatzen ${ }^{\dagger}$ \\ *Center for Fast Ultrasound Imaging, Department of Electrical Engineering, Technical University of Denmark \\ ${ }^{\dagger}$ Mads Clausen Institute for Product Innovation, University of Southern Denmark, 6400 Sønderborg, Denmark \\ Email: db@elektro.dtu.dk, jaj@elektro.dtu.dk, willatzen@mci.sdu.dk
}

\begin{abstract}
The influence of different model parameters describing a multilayer transducer model is addressed by altering each single simulation parameter within $\pm 20 \%$ in steps of $2 \%$ and by calculating the pressure and the intensity at a field point located $112 \mathrm{~mm}$ from the source. The simulations are compared with a hydrophone measured pressure pulse and intensity from a single element of a 128 element convex medical transducer. Results show that mainly the lens material and the ceramic material are of importance for errors in the pressure pulse prediction. Specifically the thickness, the density, and the stiffness constants are of significance. Among the results it is found that a $-4 \%$ change in lens stiffness yields a $6 \%$ relative error change and a $-4 \%$ change in ceramic stiffness yields a $-1.2 \%$ relative error change. When calculating intensity the piezoceramic and electronic driving circuits are of importance, where a similar change in the lens and the ceramic stiffness shows a $-0.1 \%$ and a $-12 \%$ relative error change, respectively.
\end{abstract}

\section{INTRODUCTION}

A transducer modeling principle has previously been developed and tested as a supplement to the Field II simulation software [1], [2], [3]. This modeling principle is a step towards calibrated intensity and pressure simulations using Field II [4], [5]. It was shown that the modeling principle is accurate within $0-2 \mathrm{~dB}$ for simulations on a simple piston model and a more advanced convex multilayered medical transducer [2], [6]. However, any exact prediction of the amplitude, phase, and attenuation tendency of the pressure pulses from complicated transducers is highly dependent on accurate knowledge of material constants as well as the electronic driving circuits. Such information is most often only known by manufacturers, and these may not even have an accurate estimate. This therefore influences transducer simulations [7], [8]. Also physical dimensions of the transducer, surface roughness, element cross talk, temperature, nonlinearity etc. are influencing the accuracy of the predictions. Previous studies [1], [2], [6] assumed knowledge of exact simulation parameters. However, small deviations in the predictions relative to the measured were found.

In this paper the influence of the different material parameters needed to represent a convex ultrasound transducer using the modeling principle used in [2] and [6] is investigated. The study is made by changing the different parameters of the transducer model within $\pm 20 \%$ of the values calculated from manufacturer information. The influence is studied as the error of the pressure and the intensity predictions relative to measurements.

\section{THEORY}

The model parameter study in this work is based on a 128 element convex medical transducer from BK Medical Aps. A cross section and a front view drawing in Fig. 1 illustrate how a single element of this transducer is build. A transducer element consists of a backing layer (B), a piezoceramic layer (P), a first matching layer (ML1), a second matching layer (ML2), and a lens (L) as seen in Fig. 1a. The transducer front is assumed to be lowered into water, wherefore the lens is in contact with the water (W). Fig. 1b shows a single element's front view dimensions. The transducer is assumed to be driven with a a) Side view

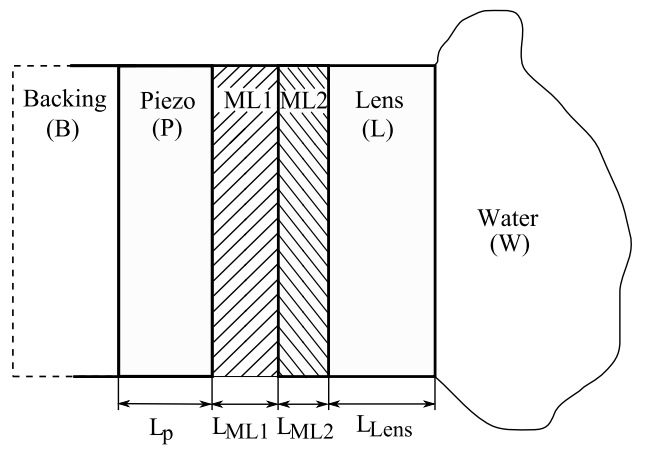

b) Front view

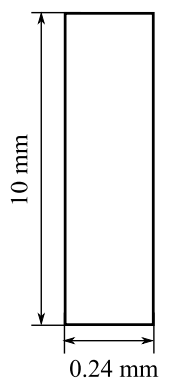

Fig. 1. Sketch of a single transducer element. a) Longitudinal cross section view. b) Front view of a single element.

transmitter unit from BK Medical placed inside our RASMUS [9] research scanner. Fig. 2 is a simplified representation of the driving electronic of such a setup. Clearly the driving circuit represented here is much less complicated than what is found in such scanner. However, by using the above simplification the complexity of the modeling is decreased.

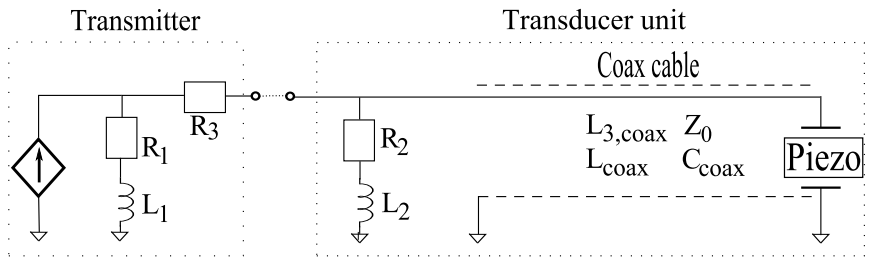

Fig. 2. Approximated electronic loading. 


$$
\begin{aligned}
& T_{B}=c_{p}^{D}\left(A_{p}+B_{p}\right)-h D, \\
& \frac{T_{B}}{Z_{B}}=\frac{c_{p}^{D}}{Z_{p}}\left(A_{p}-B_{p}\right), \\
& c_{M L 1}^{D}\left(A_{M L 1}+B_{M L 1}\right)=c_{p}^{D}\left(A_{p} e^{-\jmath k_{p} L_{p}}+B_{p} e^{\jmath k_{p} L_{p}}\right)-h D, \\
& \frac{c_{M L 1}^{D}}{Z_{M L 1}}\left(A_{M L 1}-B_{M L 1}\right)=\frac{c_{p}^{D}}{Z_{p}}\left(A_{p} e^{-\jmath k_{p} L_{p}}-B_{p} e^{\jmath k_{p} L_{p}}\right), \\
& c_{M L 2}^{D}\left(A_{M L 2}+B_{M L 2}\right)=c_{M L 1}^{D}\left(A_{M L 1} e^{-\jmath k_{M L 1} L_{M L 1}}+B_{M L 1} e^{\jmath k_{M L 1} L_{M L 1}}\right), \\
& \frac{c_{M L 2}^{D}}{Z_{M L 2}}\left(A_{M L 2}-B_{M L 2}\right)=\frac{c_{M L 1}^{D}}{Z_{M L 1}}\left(A_{M L 1} e^{-\jmath k_{M L 1} L_{M L 1}}-B_{M L 1} e^{\jmath k_{M L 1} L_{M L 1}}\right), \\
& c_{L}^{D}\left(A_{L}+B_{L}\right)=c_{M L 2}^{D}\left(A_{M L 2} e^{-\jmath k_{M L 2} L_{M L 2}}+B_{M L 2} e^{\jmath k_{M L 2} L_{M L 2}}\right), \\
& \frac{c_{L}^{D}}{Z_{L}}\left(A_{L}-B_{L}\right)=\frac{c_{M L 2}^{D}}{Z_{M L 2}}\left(A_{M L 2} e^{-\jmath k_{M L 2} L_{M L 2}}-B_{M L 2} e^{\jmath k_{M L 2} L_{M L 2}}\right), \\
& T_{W}=c_{L}^{D}\left(A_{L} e^{-\jmath k_{L} L_{L}}+B_{L} e^{\jmath k_{L} L_{L}}\right), \\
& -\frac{T_{W}}{Z_{W}}=\frac{c_{L}^{D}}{Z_{L}}\left(A_{L} e^{-\jmath k_{L} L_{L}}-B_{L} e^{\jmath k_{L} L_{L}}\right), \\
& -\jmath \omega V_{+}+-\jmath \omega V_{-}=-\jmath \omega \frac{L_{p}}{\epsilon^{S}} D-\left[h \frac{c_{M L 1}^{D}}{Z_{M L 1}}\left(A_{M L 1}-B_{M L 1}\right)-h \frac{c_{p}^{D}}{Z_{p}}\left(A_{p}-B_{p}\right)\right], \\
& -\jmath \omega A D=\frac{1}{Z_{0}} V_{+}-\frac{1}{Z_{0}} V_{-}, \\
& V(\omega)=\left(\frac{Z_{g}}{Z_{0}}+\frac{Z_{g}}{R_{2}-\jmath \omega L_{2}}+1\right) V_{+} e^{\jmath \gamma L_{\operatorname{coax}}}+\left(-\frac{Z_{g}}{Z_{0}}+\frac{Z_{g}}{R_{2}-\jmath \omega L_{2}}+1\right) V_{-} e^{-\jmath \gamma L_{\operatorname{coax}}} .
\end{aligned}
$$

Equations (1) to (13) [6] are used to model the transducer setup. The equations are to be solved for the unknown coefficients $T_{F}, T_{W}, A_{p}, B_{p}, A_{M L 1}, B_{M L 1}, A_{M L 2}, B_{M L 2}$, $A_{L}, B_{L}, D, V_{+}$, and $V_{-}$, by casting the equation system into matrix form and applying Matlab. The model assumes all layers to operate in their thickness modes only, (i.e. the 33 mode). The coefficients $Z_{B}, Z_{M L 1}, Z_{M L 2}, Z_{L}$, and $Z_{F}$ are the acoustic impedances given by $Z_{i}=\rho_{i} \mathbf{v}_{i}$, where $\rho$ and $\mathbf{v}$ are the material layer density and the speed of sound in complex form [3], respectively. The mechanical stiffness coefficients $c_{B}^{D}, c_{P}^{D}, c_{M L 1}^{D}, c_{M L 2}^{D}, c_{L}^{D}$, and $c_{W}^{D}$ are used to calculate the real valued form of the speed of sound as $v_{i}^{r}=\sqrt{c_{i_{r}}^{D} / \rho_{i}}$. The complex valued form of the velocity is $v_{i}^{r} /\left(1+\frac{j \alpha_{i} v_{i}^{r}}{\omega}\right)$, where $\alpha_{i}$ is the attenuation constant of the material [3]. The wave propagation constants $k_{P}, k_{M L 1}, k_{M L 2}$, and $k_{L}$ account for attenuation and are given by $k_{i}=\omega / \mathbf{v}_{i}$, where $\omega$ is the angular frequency. The four layers $P, M L 1, M L 2$, and $L$ have the thicknesses $L_{P}, L_{M L 1}, L_{M L 2}$, and $L_{L}$, respectively. Special constants for the ceramic are the piezoelectric coefficient $h$ and the permittivity $\epsilon^{S}$. The latter is accounting for dielectric losses through $\epsilon^{S}=\epsilon_{r}^{S}+\jmath \epsilon_{r}^{S} \tan (\delta)$, where $\epsilon_{r}^{S}$ is the real valued permittivity and $\tan (\delta)$ is the tangential loss factor [3]. The electronic network is represented with the coax cable having length $L_{\text {coax }}$, characteristic impedance $Z_{0} \cdot \gamma$ is the propagation constant defined as $\omega \sqrt{L_{3, \operatorname{coax}} C_{\operatorname{coax}}}$, where $L_{3, \text { coax }}$ and $C_{\text {coax }}$ are the cable series inductance per unit length and the shunt capacitance per unit length. The impedance $Z_{g}$ is given by $Z_{g}=R_{1}+R_{3}-\jmath \omega L_{1}$, where $R_{1}, R_{3}$, and $L_{1}$ are resistances and an inductance. $R_{2}$ and $L_{2}$ are a resistance and an inductance. The front cross sectional area, $A$, is given by the dimensions shown in Fig. 1b.

\section{MEASUREMENTS}

The measurements of the pressure field from a single transmitting element is performed by submerging the transducer into a water bath and placing a needle hydrophone in front of it at a distance of approximately $112 \mathrm{~mm}$. An Agilent MSO6014A oscilloscope was used to sample the measured pressure, and the transducer was driven at $4.0 \mathrm{MHz}$ using the RASMUS system.

\section{Simulation}

The Field II software was set up to represent the convex transducer using the command xdc_convex_focused_array. The sampling frequency was set to $400 \mathrm{MHz}$. The simulations in our previous works [2], [6] used a fixed parameter set calculated from manufacturer supplied informations. The latter parameters are used as the zero reference (ZR). All 35 parameters are altered in steps of $2 \%$ within a limit of $\pm 20 \%$ around their ZR value. When altering one parameter, the remaining parameters are held at the ZR. For each altering the root mean square (RMS) error is calcualted for the pressure and the intensity relative to the measured value. The pressure pulses are fixed in time, meaning that the cross correlation time that yields the lowest RMS error when using the ZR for simulation is aplied to all the pressure pulses where parameters differ from the ZR. To compare intensities the spatial peak pulse average is used. 


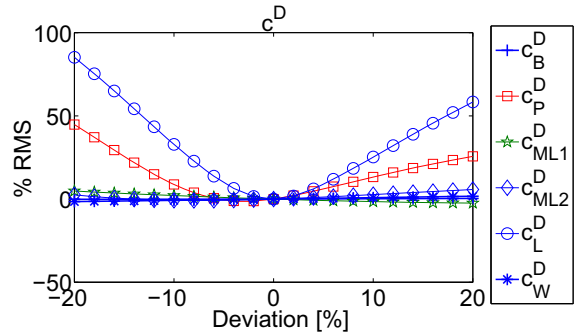

(a) Changes in the stiffness constants.

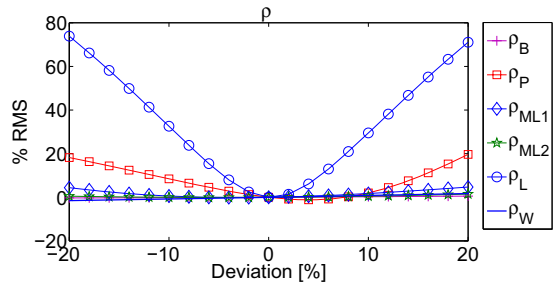

(c) Changes in the densities.

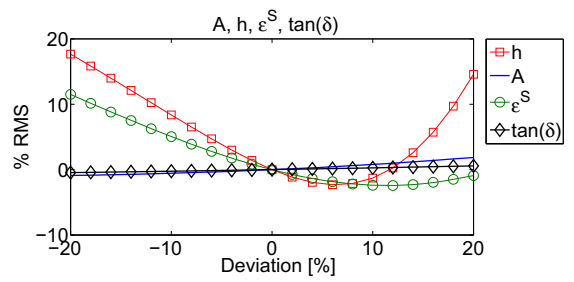

(e) Changes in the element area, $e^{S}, h$ and $\tan (\delta)$.

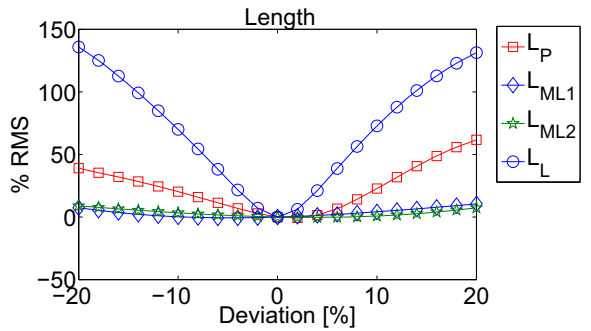

(b) Changes in the thickness of the layers.

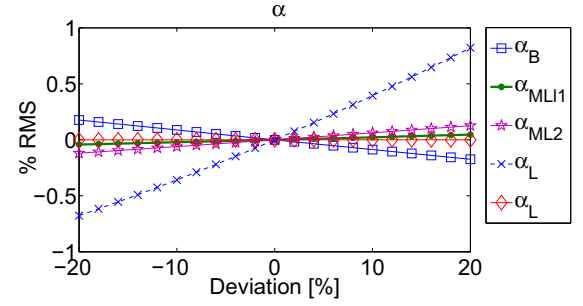

(d) Changes in the attenuation constants.

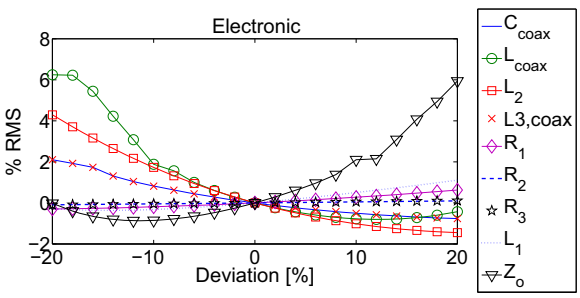

(f) Changes in the electronic components.

Fig. 3. RMS errors when simulated pressure pulses are compared with measurements.

\section{RESULts}

Fig. 3a to $3 \mathrm{~d}$ show the relative RMS errors in percent when subtracting simulated and measured pressure pulses from each other. The errors seen in the figures are all subtracted a $32.9 \%$ RMS error being the RMS error when using the ZR model values. This results initially in a $0 \%$ RMS for a $0 \%$ altering of the parameters as shown in the figures. From the figures it is clearly identified that the model is mainly sensitive to the lens (subscript L) and the ceramic (subscript P) parameters. All other components have a relatively neglectibly small affect on the error. Additionally it can be concluded, that the stiffness, $c_{i}^{D}$, the length, $L_{i}$, and the density, $\rho_{i}$, are the important parameters of the materials. Obviously these three constants affect the phase of the simulated pulse through the propagation constants $k_{i}$. The attenuation constant is seen to affect the model linearly, however, the affect is small as shown in Fig. $3 d$.

Fig. 3e shows the RMS error of the pressure pulse comparison when changes to $A, h, \epsilon^{S}$, and $\tan (\delta)$ are performed. From these results it is identified that the main factors are $h$ and $\epsilon^{S}$ which both exhibit a non linear affect on the equations. Notice that the RMS error can even be lowered by $2.3-2.5 \%$ RMS by increasing the values of these two parameters with $6-8 \%$. Changes to the area, $A$, and the ceramics electrical damping are only of slight effect. Notice, however, that for the area, $A$, only the area in (12), and not the area set by defining the geometry in the Field II software, is altered. This is done because this study investigates the sensitivity of the transducer model describing the impulse response and not the Field II surface model and/or changes in the geometry. Clearly, the error would change if the area of the Field II elements where changed as well.

The errors in Fig. $3 f$ indicate that changes in the electronic loading have an affect. However, the error is small compared to changes in the lens and the ceramic, and the affect on the model has a non linear tendency for most of the electronic parameters.

The last six plots, Fig. 4a to 4f, show the RMS intensity errors (IE). For IEs the exact phase requirements are not necessarily needed. The influencing factor is the energy of the pulse itself.

Fig. 4a to Fig. 4c reveal that the piezoceramic is affecting the error more than the lens material, which is different from the pressure pulse study. Also notice that the error is not more sensitive to lens parameters as compared to other transducer material parameters. The attenuation constant in Fig. $4 d$ is an exception albeit the influence of errors in that parameter is relatively small. Fig. 4e shows the same tendency as Fig. $3 \mathrm{e}$ hence conclusions are the same. Fig. If shows that the 


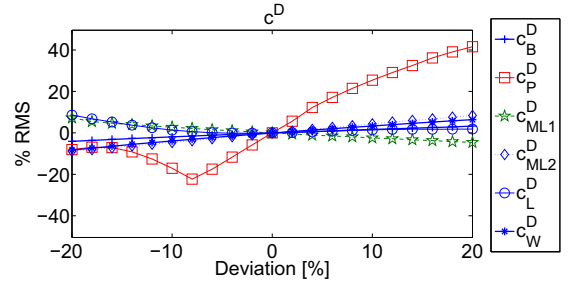

(a) Changes in the stiffness components and the intensity error.

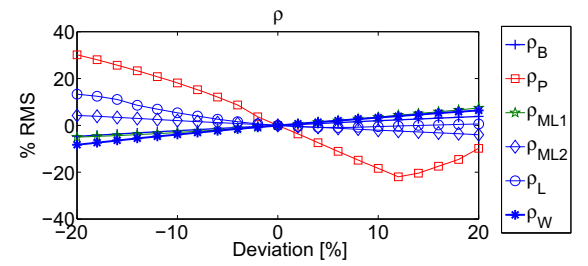

(c) Changes in the density components and the intensity error.

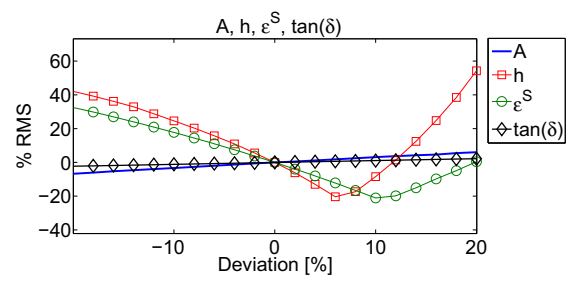

(e) Changes in the piezoelectric components and the intensity error.

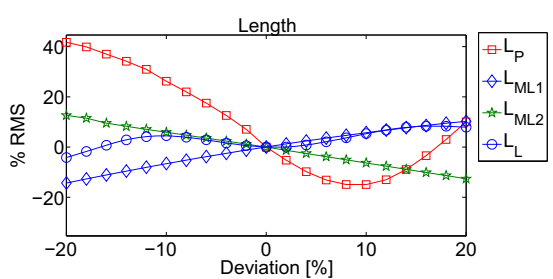

(b) Changes in the length components and the intensity error.

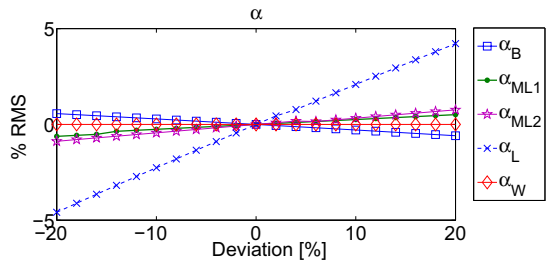

(d) Changes in the attenuation components and the intensity error.

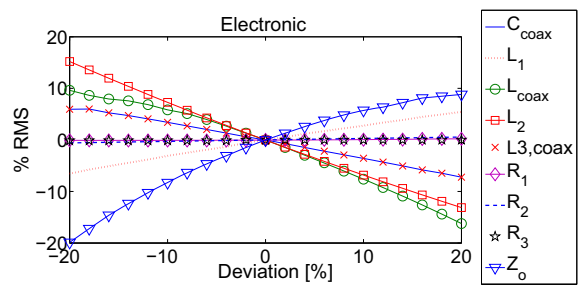

(f) Changes in the electronic components and the intensity error.

Fig. 4. RMS errors of the intensity when comparing simulation and measurements.

electronic components have increased their influences. The reason for this can be explained by the fact that the loading electronic mainly determines the clamped voltage across the piezoceramic more than influencing the phase of the pulse. This is also why it theoretically is possible to generate a zero error for the intensity with these parameters. By studying the figures quantitatively it can be found that a RMS PPE of approximately $6 \%$ for $-4 \%$ stiffness change of the $c_{L}^{D}$ and a PPE of approximately $-1.2 \%$ for a $-4 \%$ change $c_{P}^{D}$ are found. A slight error improvement is therefore achieved by changing $c_{P}^{D}$. The same study for the RMS IE is $-0.1 \%$ and $-12 \%$ for $c_{L}^{D}$ and $c_{P}^{D}$, respectively. Note also $c_{P}^{D}$ in Fig. $4 \mathrm{a}$, where a $-8 \%$ change improves the IE by $-22.3 \%$. Similar tendencies are found for $L_{P}$ and $\rho_{P}$ in Fig. 4b-c. This indicates that it is possible to approach the measured energy by changing these parameters. However, this may result in an increasing PPE.

\section{CONCLUSION}

By altering the different model parameters one at a time it is determined that for PPE calculations of simulated pressure relative to the measured the model exhibits highest sensitivity to the piezoceramic and the lens parameters. Mainly the stiffness, the thickness, and the density of these two layers are of importance. The remaining parameters were seen to have much less influence on the PPE. When comparing the RMS
IE the lens became of less significance but the piezoceramic is still influential. Also the electronic network has a significant influence on the IE.

\section{REFERENCES}

[1] D. Bæk, J. A. Jensen, and M. Willatzen, "Testing of a one dimensional model for Field II calibration," in Proc. IEEE Ultrason. Symp., Oct. 2008, pp. 1417-1420.

[2] D. Bæk, J. A. Jensen, and M. Willatzen, "Calibration of Field II using a convex ultrasound transducer," in Proc. Int. Cong. on Ultrasonics, 2009 p. Under review.

[3] M. Willatzen, "Ultrasound transducer modeling - general theory and applications to ultrasound reciprocal systems," IEEE Trans. Ultrason., Ferroelec., Freq. Contr., vol. 48, no. 1, pp. 100-112, January 2001.

[4] J. A. Jensen, "Field: A program for simulating ultrasound systems," Med. Biol. Eng. Comp., vol. 10th Nordic-Baltic Conference on Biomedical Imaging, Vol. 4, Supplement 1, Part 1, pp. 351-353, 1996 b.

[5] J. A. Jensen and N. B. Svendsen, "Calculation of pressure fields from arbitrarily shaped, apodized, and excited ultrasound transducers," IEEE Trans. Ultrason., Ferroelec., Freq. Contr., vol. 39, pp. 262-267, 1992.

[6] D. Bæk, J. A. Jensen, and M. Willatzen, "Modeling transducer impulse responses for predicting calibrated pressure pulses with the ultrasound simulation program Field II," J. Acoust. Soc. Am., p. Submitted, 2009.

[7] M. Brissaud, "Characterization of Piezoceramics," in IEEE Trans. Ultrason., Ferroelec., Freq. Contr., vol. 38, 1991, pp. 603-617.

[8] A. Ruz, A. Ramos, and J.L. San Emeterio, "Estimation of some transducer parameters in a broadband piezoelectric transmitter by using an artificial intelligence technique," in Ultrasonics, vol. 40, 2004, pp. 459-463.

[9] J. A. Jensen, O. Holm, L. J. Jensen, H. Bendsen, H. M. Pedersen, K. Salomonsen, J. Hansen, and S. Nikolov, "Experimental ultrasound system for real-time synthetic imaging," in Proc. IEEE Ultrason. Symp. vol. 2, 1999, pp. 1595-1599. 